\title{
Adaptive Power and Rate Control for Satellite Communications in Ka Band
}

\author{
Milica Stojanovic and Vincent Chan \\ Department of Aeronautics and Astronautics \\ Massachusetts Institute of Technology \\ Cambridge, MA 02139
}

\begin{abstract}
Adaptive power and rate control for efficient use of satellite channels in the Ka band are considered. Fading due to rain is modeled as a log-normally distributed, auto-regressive process with known statistics. Adaptation policies based on MMSE channel estimation are designed taking into account the effect of channel estimation errors in an optimal manner so as to satisfy the required outage probability for given fading dynamics. Analytical results show average power savings or average bit rate increase that are available from adaptive techniques. It is demonstrated that a large improvement over a non-adaptive system is available provided an appropriately designed adaptation scheme that makes use of the channel fading statistics. Finally, practical methods based on adaptive modulation level selection in a fixed-bandwidth system are evaluated analytically in terms of average bandwidth efficiency.
\end{abstract}

Index Terms-satellite communications, Ka band, rain fading, adaptive modulation, power control.

\section{INTRODUCTION}

With an increased demand for direct-to-user satellite services, Ka band has become a preferred alternative for many new systems as it offers a large bandwidth needed to support the anticipated need for capacity. While offering a solution to the problem of spectral congestion, Ka band suffers from fading due to rain (and, to a lesser degree, scintillation). Fade depths of tens of $\mathrm{dBs}$ are commonly caused by rain [1], [2].

Satellite systems are normally designed for a pre-specified outage probability, which leads to very large fixed fade margins. The consequence of such design is a wasteful use of system resources. To overcome this problem, one can either save power by reducing the transmission level during favorable channel conditions or increase the information throughput by transmitting at a higher rate. While adaptive power control is at least conceptually straightforward, adaptive rate control can be achieved by varying the bit rate, the coding rate, the modulation level, or any combination thereof. The preferred approach depends on the system optimization criterion and design constraints.

Adaptive modulation methods have been extensively studied for mobile cellular communications [3]-[5]. Power and rate adaptation policies which maximize the capacity of a given fading channel subject to a constraint on the average transmitted power were derived in [3]. Simpler methods, which use adaptive modulation-level selection and power control based on channel inversion, were shown to perform close to the optimal ones, thus providing a large improvement in spectral efficiency over the non-adaptive systems.

To implement a desired adaptation policy, the channel gain (or equivalently fading attenuation) must be known. In practice, the channel gain is not known, and an estimate must be used instead. Errors in channel estimation cause the power and rate adaptation to deviate from ideal, which results in increased average bit error rate and increased outage probability. To keep the outage probability at a pre-specified level, some margin must be introduced to account for imperfect channel estimation. The system performance is sensitive to the choice of margin, which is usually determined experimentally to match the given channel conditions [4].

In this paper, we investigate power and rate adaptation methods that are based on statistical modeling of the channel. Fading due to rain in the Ka band is well described as a slowlyvarying, log-normally distributed process. Its dynamics can be modeled as a first-order auto-regressive process, as supported by the experimental data [2]. This model gives rise to an optimal channel estimator that can be used in adaptive power and rate control. The adaptation algorithms are designed to provide the desired outage probability at a given bit error rate. To account for the channel estimation errors, a margin is introduced whose optimal value is determined analytically in terms of the statistical parameters of the fading model.

The channel model and the estimator are described in Sec.II. In Sec.III, power and rate adaptation policies are proposed for several design constraints. Analytical results are given which show the power savings and the throughput increase available from optimally designed adaptation strategies. Practical methods based on adaptive modulation level selection in a fixedbandwidth system are also considered, and evaluated analytically in terms of average bandwidth efficiency. Conclusions are summarized in Sec.IV.

\section{CHANNEL MODEL AND THE ESTIMATOR}

For a transmitted signal power $P_{T}$, the received signal power, $P_{R}=P_{T} G$, is given in terms of the channel gain $G$ which is modeled as log-normally distributed. Thus, the random variable $X=\ln G$ is normally distributed with some mean $m$ and variance $\sigma^{2}$. The relevant parameters of the channel gain $G$ are its mean, $\bar{G}=e^{m+\sigma^{2} / 2}$, and the mean of its inverse $F=1 / G, \bar{F}=e^{-m+\sigma^{2} / 2}$

The dynamics of rain fading in the Ka band are generally described by an auto-regressive moving-average (ARMA) 
model [1]. A special case of this model is the first-order autoregressive (AR1) model, which was found in [2] to provide a good match with experimental data. The AR1 model represents the fading process in discrete time as

$$
\begin{aligned}
\Delta X(n) & =a \Delta X(n-1)+W(n) \\
X(n) & =m+\Delta X(n)
\end{aligned}
$$

where $n$ indicates time taken is steps $T_{d}$, and $W(n)$ is zeromean, white Gaussian process, which is independent of $X(n-$ 1 ). The parameter $a$ is the one-step correlation coefficient:

$$
a=\frac{E\{\Delta X(n) \Delta X(n-1)\}}{\sigma^{2}}=\frac{R_{\Delta X}\left(T_{d}\right)}{R_{\Delta X}(0)}
$$

where $R_{\Delta X}(\Delta t)$ represents the autocorrelation function of the underlying continuous-time process. The power spectral density is given by the Fourier transform

$$
S_{\Delta X}(f)=F\left[R_{\Delta X}(\Delta t)\right]=\frac{\sigma^{2} / \pi f_{d}}{1+\left(f / f_{d}\right)^{2}}
$$

where $2 f_{d}$ is the $3-\mathrm{dB}$ bandwidth or the Doppler spread of the fading process. The parameter $a$ can be expressed as

$$
a=e^{-\omega_{d}\left|T_{d}\right|}
$$

where $\omega_{d} T_{d}=2 \pi f_{d} T_{d}$ is termed the normalized fading rate.

If the coefficient $a$ is known, the minimum mean squared error (MMSE) channel estimate $\hat{X}(n)$, obtained upon observing the delayed channel gain $X(n-1)$ is given by

$$
\begin{aligned}
\Delta \hat{X}(n) & =a \Delta X(n-1) \\
\hat{X}(n) & =m+\Delta \hat{X}(n)
\end{aligned}
$$

The estimation error, $E(n)=X(n)-\hat{X}(n)$, is orthogonal to the estimate $\Delta \hat{X}(n)$. The variance of the error is $\sigma_{E}^{2}=$ $\left(1-a^{2}\right) \sigma^{2}$, and the variance of the estimate is $\sigma_{\hat{X}}^{2}=a^{2} \sigma^{2}$.

The parameters $m, \sigma^{2}$ and $a$ can be estimated from measurements. In what follows, we shall assume that these parameters are known. Such an assumption is deemed to be more realistic than the assumption of a perfectly known realization of the channel gain $G$.

\section{POWER AND RATE ADAPTATION}

In this section we consider a hypothetical transmission scheme which uses a single modulation method (QPSK for instance) and in which power or rate can be adjusted continuously. We first examine the conventional, fixed margin link design. Then, we address adaptation strategy in two cases: when perfect knowledge of the channel gain is available, and when only an estimate is available. The design constraint is a given outage probability, $P_{\text {out }}$ at a specified bit error probability $P_{e 0}$. In each case, we evaluate the average improvement (average power savings for power adaptation or average rate increase for rate adaptation).

Let the AWGN bit error probability for the chosen modulation method be given by a known function $P_{e}=P_{e}[\gamma]$, where $\gamma=P_{T} G / N_{0} R$ is the SNR, given in terms of the received power $P_{T} G$, the symbol rate $R$ and the double-sided power spectral density $N_{0} / 2$ of the $\mathrm{AWGN}$ at the receiver input. The outage probability is the probability that the bit error rate is greater than the specified required value $P_{e 0}$ : $P_{\text {out }}=P\left\{P_{e}>P_{e 0}\right\}=P\left\{\gamma<\gamma_{0}\right\}$, where $\gamma_{0}=P_{e}^{-1}\left[P_{e 0}\right]$.

When the transmitter power is constant, the outage probability is given by $P_{\text {out }}=P\left\{G<N_{0} R \gamma_{0} / P_{T}\right\}=P\left\{G<G_{\text {out }}\right\}$. For the assumed log-normal fading, we have that

$$
P_{\text {out }}=Q\left[\frac{m-\ln G_{\text {out }}}{\sigma}\right]
$$

where $Q$ denotes the Q-function, $Q(x)=1 / 2 \operatorname{erfc}(x / \sqrt{2})$. The outage threshold is thus defined as

$$
G_{\text {out }}=e^{m-\sigma Q^{-1}\left[P_{\text {out }}\right]}
$$

If the power needed to achieve $P_{e 0}$ in the absence of fading $(G=1)$ is $P_{T}=P_{T 0}=N_{0} R \gamma_{0}$, when there is fading, the power needed to achieve $P_{e 0}$ with outage $P_{\text {out }}$ is $P_{T}=P_{T 0} / G_{\text {out }}$. The factor $1 / G_{\text {out }}$ represents the needed fixed margin. For example, with $\left(m, \sigma^{2}\right)=(-0.5,1)$, which give $\bar{G}=1$, and for $P_{\text {out }}=0.01$, which is a figure representative of commercial satellite systems, the margin is $10 \log \left(1 / G_{\text {out }}\right)=12.3 \mathrm{~dB}$. This is a high margin to use in a system where power is at premium.

\section{A. Adaptive Power Control}

In an ideal adaptive power control scheme, the transmitted power can be varied in accordance with the known fading gain $G$, so that the SNR is kept at the value $\gamma_{0}: P_{T}=P_{T 0} / G$. The bit error rate is constant, $P_{e 0}$, and there is no outage. The average power consumed in this manner is $\overline{P_{T}}=P_{T 0} \bar{F}=$ $P_{T 0} e^{-m+\sigma^{2} / 2}$.

For application to satellite systems, it is more reasonable to consider the case of limited power. In this case, an appropriate adaptation policy is

$$
P_{T}=\left\{\begin{array}{cc}
P_{T 0} / G, & P_{T 0} / G \leq P_{T \max } \\
0, & \text { otherwise }
\end{array}\right.
$$

The outage now occurs whenever the power is turned off: $P_{\text {out }}=P\left\{G<P_{T 0} / P_{\text {Tmax }}\right\}$. Thus, to meet the required $P_{\text {out }}$, one has to use $P_{\text {Tmax }}=P_{T 0} / G_{\text {out }}$, where $G_{\text {out }}$ is given in (7). The average transmitted power with this adaptation strategy is evaluated to be

$$
\overline{P_{T}}=P_{\text {Tmax }} G_{\text {out }} e^{-m+\sigma^{2} / 2} Q\left[\sigma-\frac{m-\ln G_{\text {out }}}{\sigma}\right]
$$

which is less than the power needed with fixed margin.

When the channel is not known, an estimate $\hat{G}$ is used in place of the true gain $G$. A continuous power adaptation algorithm is then given by

$$
P_{T}=P_{T 0} K / \hat{G}
$$

The constant $K$ represents a new margin (hopefully lower than $1 / G_{\text {out }}$ ) that must be introduced to ensure that the discrepancy 
between the estimated and the true channel gain does not lead to outage. In particular, we determine $K$ such that the requirement on $P_{\text {out }}$ is satisfied. With the above power adaptation, the outage probability is

$$
P_{\text {out }}=P\{K G / \hat{G}<1\}=Q\left[\frac{\ln K}{\sqrt{1-a^{2}} \sigma}\right]
$$

where the last equality follows from the properties of the estimator described in Sec.II. Comparing this result with the outage probability defined in (6), we obtain the optimal value of $K$ :

$$
K=\left[e^{m} / G_{\text {out }}\right]^{\sqrt{1-a^{2}}}
$$

The average power is now given by

$$
\overline{P_{T}}=P_{T 0} K e^{-m+a^{2} \sigma^{2} / 2}
$$

We note that as $a \rightarrow 1$ (very slow variation), $P_{T} \rightarrow$ $P_{T 0} e^{-m+\sigma^{2} / 2}$, the value obtained for the case of known fading. As $a \rightarrow 0$ (very fast fading), $P_{T} \rightarrow P_{T 0} / G_{\text {out }}$, the value which equals the fixed margin case. Thus, in the worst case, this scheme uses as much power as the fixed-margin non-adaptive scheme. However, the worst case (infinitely fast fading) is not a realistic one.

Fig. 1 shows the average power normalized by that required with fixed margin, $\overline{P_{T}} /\left(P_{T 0} / G_{\text {out }}\right)$. The power is shown as a function of the normalized fading rate, expressed through the factor $1-a$. This scale was chosen for the sake of completeness, but we keep in mind that for all practical situations $\omega_{d} T_{d}<<1$, and, thus, $1-a \approx \omega_{d} T_{d}$. The results are presented for an example channel $\left(m, \sigma^{2}\right)=(-0.5,1)$. The design is based on the required $P_{\text {out }}=0.01$. Solid line at approximately $-8 \mathrm{~dB}$ represents the average normalized power needed when the channel is perfectly known, i.e., it shows the maximal savings obtained with respect to the fixed margin case.

Solid curve shows the average power used when $K$ is chosen optimally as in (12) to match the fading rate of the channel. With an increase in fading rate, the average power increases from the value needed with perfect channel estimation to that needed in a non-adaptive system with fixed margin. Typical values of Doppler spread for the $\mathrm{Ka}$ band are below $1 \mathrm{~Hz}$. For a LEO system, with a round-trip delay on the order of 10 $\mathrm{ms}$, the normalized fading rate will be at most $\omega_{d} T_{d} \sim 10^{-2}$. Thus, although there will be some power increase due to the inevitable delay in channel observation, substantial savings are available from adaptive power control.

Dashed curves show the power used by an adaptive system in the case when $K$ is not chosen in accordance with the fading rate, but is kept fixed regardless of the channel dynamics. The curves, labeled $K_{i}, i=0,1$, etc. correspond to the cases of $K$ evaluated using (12) at $a=1-10^{-i}$. The curve labeled $K=1$ shows the power that would be needed if no margin were used, i.e. with power adapted as $P_{T}=P_{T 0} / \hat{G}$. While the average power in this case stays below the power needed with perfectly known channel, the requirement on outage probability is not met. This fact is demonstrated in Fig.2, which shows the outage probability as a function of the fading rate parameter $1-a$ for the choices of $K$ corresponding to those of Fig.1. The choice $K=1$ results in an unacceptable outage

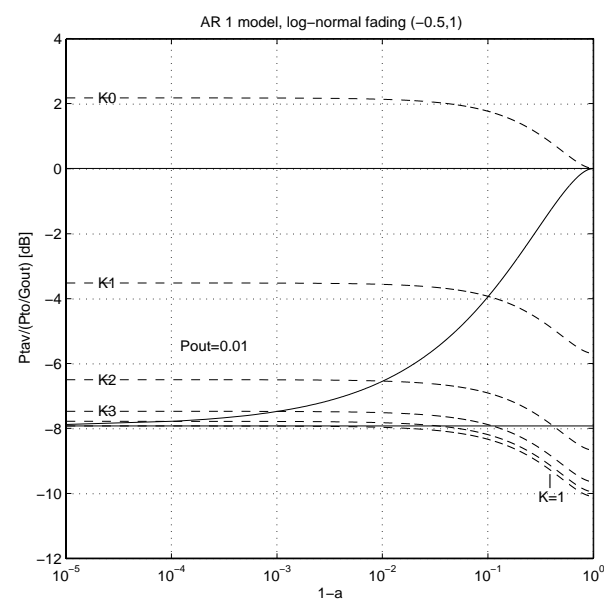

Fig. 1. Average normalized power used by the system with adaptive power control, as a function of fading rate parameter $1-a$. Instantaneous power is varied in accordance with the channel estimate. Solid line corresponds to the case of perfectly known channel. Solid curve corresponds to an optimal adaptation policy which ensures the required outage probability $P_{\text {out }}$ at every fading rate.

probability (1/2). For example, if $K=K_{2}$ were calculated assuming a fading rate of $1-a=10^{-2}$, then for an actual rate less than $10^{-2}$, the outage probability would remain below the required value 0.01 , but the average power would be higher than necessary. For the actual fading rate greater than $10^{-2}$, the outage probability would exceed the required value. The outage probability curves show high sensitivity to the choice of $K$. A constant value $P_{\text {out }}=0.01$ is attained at every fading rate if $K$ is chosen optimally as in (12).

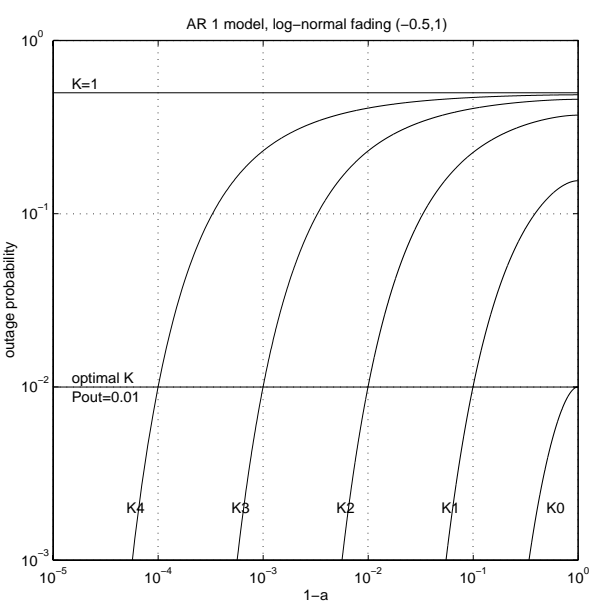

Fig. 2. Outage probability as a function of fading rate parameter $1-a$ for several power adaptation policies.

To complete the analysis of adaptive power control, we now investigate the case of estimated channel gain and limited power. In this case, a candidate adaptation algorithm is

$$
P_{T}=\left\{\begin{array}{cc}
P_{T 0} K / \hat{G}, & \hat{G} \geq G_{\text {out }} \\
0, & \text { otherwise }
\end{array}\right.
$$

Alternatively, one may impose a limitation on the maximal power, in which case the on/off decision to transmit would be 
made based on the instantaneous power, $P_{T 0} K / \hat{G}$, rather than the channel gain estimate, $\hat{G}$. While it is possible to analyze both algorithms, we shall focus on the first one.

Following the previous approach, we determine $K$ for which the requirement on $P_{\text {out }}$ is met. The outage probability is now

$$
\begin{aligned}
P_{\text {out }} & =P\left\{\hat{G}<G_{\text {out }}\right\}+P\left\{\hat{G} \geq G_{\text {out }}\right\} \\
& \times P\left\{K G / \hat{G}<1 \mid \hat{G} \geq G_{\text {out }}\right\}
\end{aligned}
$$

where the first term represents outage due to shut-off $\left(P_{T}=0\right)$ and the second term represents outage due to estimation errors. Using the orthogonality property of the estimator of Sec.II, the above expression simplifies to

$$
\begin{gathered}
P_{\text {out }}=Q\left[\frac{m-\ln G_{\text {out }}}{|a| \sigma}\right]+ \\
\left(1-Q\left[\frac{m-\ln G_{\text {out }}}{|a| \sigma}\right]\right) Q\left[\frac{\ln K}{\sqrt{1-a^{2}} \sigma}\right]
\end{gathered}
$$

From this expression, it follows that the optimal value of $K$ is given by

$$
\begin{array}{r}
K=\left[\exp \left(\sigma Q^{-1}\left(\frac{P_{\text {out }}-P_{\text {off }}}{1-P_{\text {off }}}\right)\right)\right]^{\sqrt{1-a^{2}}} \\
\text { where } \quad P_{\text {off }}=Q\left[\frac{m-\ln G_{\text {out }}}{|a| \sigma}\right]
\end{array}
$$

and $G_{\text {out }}$ is defined in (7). The average power consumed by this adaptation scheme for any value of $K$ is

$$
\overline{P_{T}}=P_{T 0} K e^{-m+a^{2} \sigma^{2} / 2} Q\left[|a| \sigma-\frac{m-\ln G_{\text {out }}}{|a| \sigma}\right]
$$

Using the optimal value (18) for $K$, we can look at two extreme cases. For $a \rightarrow 1$ (very slow fading), outage occurs mostly due to shut-off, $P_{\text {off }} \rightarrow P_{\text {out }}$, and the average power approaches the value (9) obtained with perfectly known channel and constrained power. As $a \rightarrow 0$ (very fast fading), we find that $P_{\text {off }} \rightarrow 0$, and $\overline{P_{T}} \rightarrow P_{T 0} / G_{\text {out }}$, the value found in the non-adaptive system design based on fixed margin, or, equivalently, the maximal power needed by ideal adaptation to achieve the required $P_{\text {out }}$.

Fig. 3 shows the normalized power, $\overline{P_{T}} /\left(P_{T 0} / G_{\text {out }}\right)$ as a function of the fading rate parameter $1-a$. Solid line represents the average power consumed in the ideal case of known fading. When channel estimation is employed, $K$ is evaluated for each fading rate as in (18). It has to be noted that with on/off control based on comparing the estimate $\hat{G}$ to the threshold $G_{\text {out }}$, the maximal power needed to achieve $P_{\text {out }}$ varies with fading rate: $P_{T \max }(a)=P_{T 0} K / G_{\text {out }}$. Thus, $K$ represents a factor by which the maximal power increases with respect to the value $P_{T \max }=P_{T 0} / G_{\text {out }}$ needed with ideal channel estimation. In the example considered, for fading rates below $10^{-2}$, this factor stays below 1.5. Hence, even with constrained maximal power, which is a necessary design assumption for a satellite system, efficient power usage is possible.

\section{B. Adaptive Rate Control}

An alternative approach to adaptive power control is adaptive rate control. In this case, the power is kept constant,

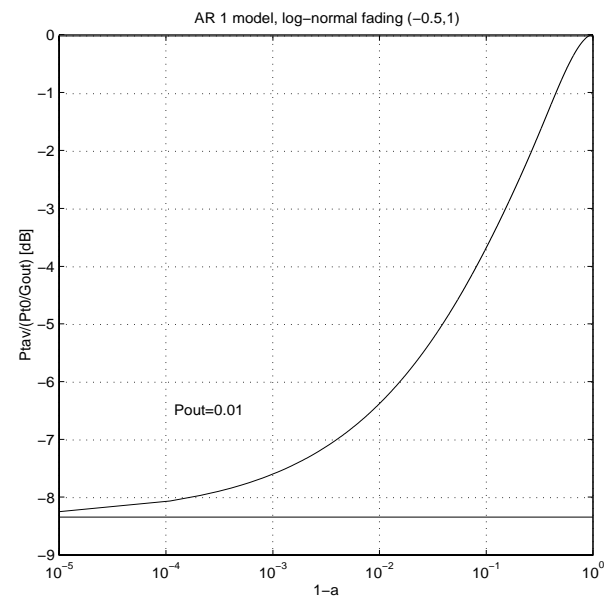

Fig. 3. Average normalized power used by the system with adaptive power control and constrained power. When transmitting, the instantaneous power is varied in accordance with the channel estimate. The required outage probability $P_{\text {out }}$ is met at every fading rate. Solid line corresponds to the case of perfectly known channel.

but the rate is varied in accordance with the channel fading. Mathematically, the two approaches are analogous. Practically, rate adaptation offers a higher average information throughput rather than power savings. For this reason, it may be the preferred method for satellite systems with constrained power.

Below we consider a hypothetical case of continuous rate variation. In practice, transmission rate can be changed in discrete steps, as allowed by the particular hardware implementation.

Ideally, rate control results in a constant received SNR $\gamma_{0}$ which suffices to provide the required bit error probability $P_{e 0}=P_{e}\left[\gamma_{0}\right]$. This situation can be realized if the channel gain is completely known. Then, we wish to have $P_{T} G / N_{0} R=\gamma_{0}$, i.e. $R=P_{T} G / N_{0} \gamma_{0}=R_{0} G$, where $R_{0}$ is the rate corresponding to a non-fading $(G=1)$ channel. There is no outage as the bit error probability always stays at $P_{e 0}$. The average rate achievable in this manner is $\bar{R}=R_{0} \bar{G}=R_{0} e^{m+\sigma^{2} / 2}$.

When the channel is not completely known, the rate may be adjusted as

$$
R=R_{0} \hat{G} / K
$$

where, as before, $K$ is a margin that accounts for the discrepancy between the estimate and the true channel. We select $K$ to meet the required outage probability: $P_{\text {out }}=P\left\{P_{T} G / N_{0} R<\right.$ $\left.\gamma_{0}\right\}=P\{K G / \hat{G}<1\}$. Thus, with the channel estimator based on the AR1 model, we have that $K$ should be chosen as in (12). The average rate achieved in this manner is

$$
\bar{R}=\frac{R_{0}}{K} e^{m+a^{2} \sigma^{2} / 2}
$$

Fig.4 shows the average rate as a function of the fading parameter $1-a$. For very slow fading, near-perfect estimation is possible, and $\bar{R} \rightarrow R_{0} \bar{G}$. As the fading becomes faster, errors in channel estimation cause the average rate to decrease. Ultimately, as fading becomes too fast to track, $\bar{R} \rightarrow R_{0} G_{\text {out }}$. This value equals the rate that a non-adaptive, fixed-rate system with fixed power $P_{T}$ would use to achieve the design goal of 
outage $P_{\text {out }}$ at bit error probability $P_{e 0}$. It is analogous to the fixed margin case. Adaptive rate control yields an increase in the average rate over the non-adaptive system by a factor whose value depends on the fading rate. This value is highest when the channel is perfectly known (or, equivalently, the fading is slow enough that near-perfect estimation is possible), and is equal to $\bar{G} / G_{\text {out }}$. Due to estimation errors, the average rate descreases, but still remains greater than in a non-adaptive system. For example, at $\omega_{d} T_{d}=0.01$, the average achievable rate is 12 times greater than if no rate control were used, and only 1.4 times lower than in the case of perfectly known fading. Hence, even in the presence of imperfect fading estimation, a substantial improvement is possible. This fact motivates the search for a practical rate control strategy.

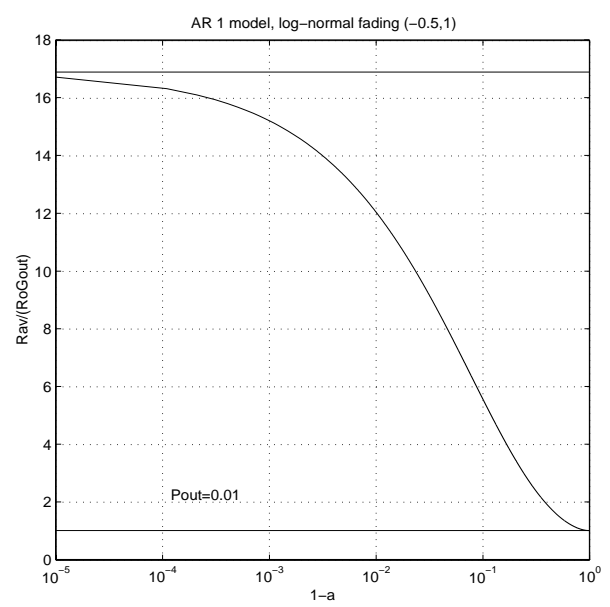

Fig. 4. Average transmission rate as a function of fading parameter $1-a$. Rate adaptation algorithm is designed to meet the required outage probability Pout. Average transmission rate is normalized by $R_{0} G_{\text {out }}$, the value needed to meet the design requirement in a non-adaptive system. Maximal average rate achieved for known channel is $R_{0} \bar{G}$.

Changing the actual transmission rate is inconvenient in practice because it requires adjustments to filters and synchronization hardware. It is much more convenient to use a fixed transmission rate, while changing the number of bits per symbol, i.e, the modulation level.

Satellite channels are characterized by the amplifier nonlinearity, and for this reason, practical systems rarely use modulation levels higher than 4. An experimental design [6] focuses on 4, 8 and 16 PSK modulation for a Ka band system. Using a higher modulation level in a constellation with varying phase and amplitude (i.e. MQAM) requires special care of nonlinear distortion. Constellation predistortion, signal predistortion, or receiver-based equalization/detection methods must be used. A conceptual design of a 16 QAM system for use in satellite channels is given in [7].

Below, we consider system design based on adaptive modulation level selection. Selection is made in such a manner that the requirement on outage probability $P_{\text {out }}$ for a given bit error rate $P_{e 0}$, is satisfied. We focus on linear system operation, and investigate the average bandwidth-efficiency that is available from adaptive modulation selection.

Let us assume that the system selects from $I$ available levels described by $k_{1}, k_{2}, \ldots k_{I}$ bits per symbol of a given modu- lation method, such as MPSK or MQAM. Their respective probabilities of error are given by known functions $P_{e, i}(\gamma)$, where $\gamma=P_{T} G / N_{0} R$ is the SNR obtained with the symbol rate $R$, which is equal for all modulation methods.

Using the outage probability as the design criterion, we first determine the SNR regions in which each of the modulation methods is to be used. In particular, the selection rule is the following:

- when $G<G_{\text {out }}=G_{0}$, nothing is transmitted (outage)

- when $G \in\left[G_{i-1}, G_{i}\right)$, method $i$ is selected $(i<I)$

- when $G \geq G_{I-1}$, method $I$ is selected $\left(G_{I}=\infty\right)$

The thresholds are evaluated to meet the design requirements $P_{\text {out }}$ and $P_{e 0}$ :

$$
G_{i}=G_{o u t} \frac{P_{e, i+1}^{-1}\left(P_{e 0}\right)}{P_{e, 1}^{-1}\left(P_{e 0}\right)}
$$

where $G_{\text {out }}$ is given in (7) as before. It may be noted that instead of shutting off the transmission when $G<G_{\text {out }}$, one can continue to transmit using the first method. This will increase the average bandwidth efficiency, but only slightly for a reasonably low outage probability. Power constraints, however, dictate that on/off control should be used.

The probability of each modulation method is evaluated under the assumption of log-normal fading:

$$
P_{i}=Q\left(\frac{m-\ln G_{i}}{\sigma}\right)-Q\left(\frac{m-\ln G_{i-1}}{\sigma}\right)
$$

The average number of bits per symbol is now obtained as

$$
\overline{R_{b}} / R=\sum_{k=k_{1}}^{k_{I}} k_{i} P_{i}
$$

A numerical example is in order to show the average bandwidth efficiency improvement available through adaptive modulation level selection. We assume the fading model with $\left(m, \sigma^{2}\right)=(-0.5,1)$ as before, and a design based on $P_{\text {out }}=$ $0.01, P_{e 0}=10^{-6}$.

MPSK and MQAM, with $M=2^{k_{i}}$ are considered. The bit error rate of a Gray-coded MPSK is approximated by [8]

$$
P_{e, i}(\gamma) \approx \frac{1}{k_{i}} 2 Q \sqrt{2 \gamma \sin ^{2}\left(\frac{\pi}{2^{k_{i}}}\right)}
$$

The approximation is valid for any $M$ and high SNR. The bit error probability of a Gray-coded square MQAM can be approximated by [3]

$$
P_{e, i}(\gamma) \approx \frac{1}{5} \exp \left(-\frac{3}{2} \frac{\gamma}{\left(2^{k_{i}}-1\right)}\right)
$$

The approximation is valid for $M \geq 4$ and SNR between $0 \mathrm{~dB}$ and $30 \mathrm{~dB}$. Results are summarized in Tables I and II.

As it can be expected, average bandwidth efficiency increases as more modulation levels are available for selection. However, there is an effect of diminishing returns. For the examples presented, addition of the next higher level (choosing from five methods) negligibly increases the average bandwidth efficiency of both MPSK and MQAM. 


\begin{tabular}{|l|l|}
\hline$k_{1}, \ldots k_{I}$ & $\overline{R_{b}} / R$ \\
\hline 2,3 & 2.85 \\
\hline $2,3,4$ & 3.28 \\
\hline $2,3,4,5$ & 3.34 \\
\hline
\end{tabular}

TABLE I

Average number of bits per symbol with MPSK.

\begin{tabular}{|l|l|}
\hline$k_{1}, \ldots k_{I}$ & $\overline{R_{b}} / R$ \\
\hline 2,4 & 3.51 \\
\hline $2,4,6$ & 3.98 \\
\hline $2,4,6,8$ & 4.01 \\
\hline
\end{tabular}

TABLE II

Average number of bits per symbol with MQAM.

Selecting from a number of QAM methods gives higher average bit rate than selecting from the same number of PSK methods. For example, selecting from 4PSK, 8PSK and 16PSK gives on the average 3.28 bits/symbol. Selecting from 4QAM, 16QAM and 64QAM gives $3.98 \mathrm{bits} / \mathrm{symbol}$. (It should be noted that $8 \mathrm{QAM}$ is unfairly left out simply because of the lack of a closed-form expression for the bit error rate, which by no means should be the case in a practical system design.) It also has to be pointed out that comparison is made on the basis of equal power $P_{T}$ in a linear system. To ensure linearity in a practical system, MQAM requires a greater back-off than MPSK.

Finally, it has to be emphasized that a practical system will use channel coding. Thus, appropriate bit error probability curves for various coding schemes must be used when evaluating the average bandwidth efficiency improvement of candidate modulation/coding schemes. In addition to modulation level and code selection, an efficient system design can incorporate power control based on the knowledge of channel statistics. In a practical system, channel estimate will be used to implement the modulation selection rule. For each selected modulation, a power margin can be designed in a manner analogous to that described in Sec.III-A.

\section{SUMMARY AND CONCLUSION}

We have addressed the problem of adaptive modulation for satellite communications in the Ka band. Rain attenuation in this band leads to a large fixed link margin if a pre-specified outage probability is to be met. Adaptive power control and rate control offer the possibility to efficiently use the system resources, by either saving the power or increasing the information throughput during the time when channel conditions are favorable.

An analytical approach was proposed to determine an adaptation policy when fading is modeled as a log-normally distributed process with dynamics described by a first-order autoregressive model. MMSE estimate of the channel gain is used to adaptively control the power or the rate. The adaptation scheme is designed to meet the required outage probability. To do so, a margin is introduced whose value is obtained analytically in terms of statistical parameters of the fading process. Results for the average power and transmission rate quantify the potential improvement offered by these techniques. It is demonstrated that a large improvement over a non-adaptive system is available provided an adaptation scheme which takes into account the effect of channel estimation errors. Further research in this area will concentrate on more sophisticated models of the fading process, such as those with multiple states.

In practice, it is more convenient to vary the modulation level than the actual transmission rate. The goal in doing so is to maximize the average bandwidth efficiency. For a given set of modulation methods, we determined a selection rule which satisfies the outage probability requirement in conditions of log-normal fading. The results show that although MPSK provides lower average bandwidth efficiency than MQAM, the loss is not severe. For a practical system, both methods should be considered taking into account the the system nonlinearity.

\section{REFERENCES}

[1] B.Gremont, M.Filip, P.Gallois and S.Bate, "Comparative analysis and performance of two predictive fade detection schemes for Ka-band countermeasures," IEEE J. Select. Areas Commun., vol.17, pp.180192, Feb. 1999.

[2] P.Choi, "Channel Prediction and Adaptation over Satellite Channels with Weather-Induced Impairment,” M.S. Thesis, M.I.T., Cambridge, MA, May 2000.

[3] A.Goldsmith and S-G.Chua, " Variable-rate variable-power MQAM for fading channels," IEEE Trans. Commun., vol. 45, pp.1218-1230, Oct. 1997.

[4] T.Ue, S.Sampei, N.Morinaga and K.Hamaguchi, "Symbol rate and modulation level-controlled adaptive modulation/TDMA/TDD system for high-bit-rate wireless data transmission," IEEE Trans. Vehic. Technology, vol.47, pp.1134-1147, Nov. 1998.

[5] C.H.Lim and J.Cioffi, "Performance of the adaptive rate MQAM with on/off power control," IEEE Commun. Letters, vol.5, pp.16-18, Jan. 2001.

[6] A.Malygin, M.Filip and E.Vilar, "Development of a digital modem for adaptive countermeasure VSAT system at Ka-band," in Proc. Intl. Conf. Satellite Commun. ICSC'96, vol. 3, pp.133 -139

[7] R.DeGaudenzi and M.Luise, "Analysis and design of an all-digital demodulator for trellis coded 16-QAM transmission over a nonlinear satellite channel," IEEE Trans. Commun., vol.43, pp.659-667, Feb/Mar/Apr. 1995.

[8] J.Proakis, "Digital Communications," McGraw Hill '01 (fourth ed.) 